\title{
Choice of a suitable e-nose output variable for the continuous monitoring of an odour in the environment.
}

\author{
Nicolas, J., Romain, A.C, Monticelli, D., Maternova, J. and André, Ph. \\ Fondation Universitaire Luxembourgeoise \\ Department "Environmental Monitoring" \\ Avenue de Longwy, 185 \\ B 6700 ARLON -(BELGIUM)
}

\section{Introduction}

Among the potential application areas for electronic noses, the monitoring of our environment constitutes a real challenge. The rigorous experimental conditions which apply in the laboratory are no longer usable in the field. Particularly, the input odour can't be considered as a well-defined variation of the gaseous ambience with respect to clean air. "In the field of environmental monitoring, the background is an ever-changing chemical mixture against which we want to detect the rise of a particular odour - although the exact profile of that rise is both unknown and variable" (Gardner and Bartlett, 1999).

There is thus a need to develop instruments able to monitor continuously the odours emerging from factories, from sewage farms or from landfills sites, in order to assess the importance of public nuisance, or to control in real time the odour abatement techniques.

The department "Environmental Monitoring" at F.U.L. (Arlon, Belgium) is active in the adaptation of the electronic nose principle to discriminate some environmental odours, if possible directly in the field, and to monitor them continuously.

Research aims at improving the portability and the user-friendliness of the instrument, by trying to supply the information actually requested by the final user in the field, i.e. either the scientific operator, or the supervisor of the odorous process (Romain, Nicolas et al., 1997, 1999, 2000).

The present paper goes in the same direction by testing some practical solutions to reach those objectives.

Three main questions are put.

- How to simplify the apparatus, in order to lower its price and to make it more easy to use in the field?

- Which is the function most adapted to classify the various sources, and which allows to make a decision in real time?

- What kind of signal may be used to monitor continuously the odour "intensity"?

\section{Material and methods}

An array of tin-oxide sensors (either TGS from Figaro Company or multisensors from Microsens) is used in different configurations. The ambient air is either sampled around environmental sources (landfill, urban waste composting facilities, ...) and transferred to the laboratory system, or directly transferred to the sensor chamber in the mobile detector, usable directly in the field. 
Various experiments, made under various conditions, led to the formulation of some practical advice concerning the design and the use of a field e-nose.

The majority of the results come however from a case study related to the odour generated by a landfill. Two kinds of odours are perceived by the neighbouring population : either the one of the fresh refuse (esters, sulphur organic compounds, solvents, ...), or the one of the biogas generated by the decomposition of the organic matter under anaerobic conditions (trace elements, such as $\mathrm{H}_{2} \mathrm{~S}, \mathrm{NH}_{3}$ and some VOC's in a mixture essentially composed of odourless compounds: methane and carbon dioxide).

Measurements are chiefly made with a mobile electronic nose constituted of 6 TGS sensors in an aluminium box $\left(100 \mathrm{~cm}^{3}\right)$. A constant gas flow rate of $150 \mathrm{ml} / \mathrm{min}$ is provided by a small pump, and the system operates by a series of cycles alternating 5 minutes of pure air (sampled in a Tedlar bag from an air generator in the lab, prior to moving in the field) and 5 minutes of odorous gas transferred directly into the sensor chamber. The whole system is powered by a $12 \mathrm{~V}$ battery.

The operator performs measurements at some different locations on the landfill area : either in the vicinity of fresh waste, sometimes when the trucks pour out the refuse, sometimes when the waste is at rest, or at various distances from a landfill gas extraction well. At each location, he notes his feeling of odour intensity on a 4 level scale. A total of 141 observations were carried out with that procedure : 69 around "fresh waste" (including 21 observations with intensity 0), and 72 around "biogas" (including 24 zero-intensity observations).

The 6 sensor signals are recorded in a data logger and they are off-line processed by Statistica or Matlab procedures, but the calibrated model is also tested for further on-line validation in the field.

Indeed, the final goal, corresponding to the wish of the landfill manager, should be to use continuously the calibrated model in the field in order to control the atomisation of the bad odour neutralisation product.

\section{Results and discussion}

\section{Simplification of the apparatus towards a mobile and user-friendly detector.}

- The test of various pre-processing data algorithms pointed out that the use of pure reference air could be avoided (i.e. the use of gas bottle or high performance filters), as long as the sensors are allowed to periodically regenerate in the presence of ambient air (Romain et al., 2000; Nicolas et al., 2000). That conclusion is drawn both for the "mobile detector" and for the laboratory system : neglecting the reference to the base line does not affect the classification of observations per source of odour.

- For the mobile detector, two types of operation are compared : the sensor array in static contact with ambient air and the use of a controlled gas flow system to transfer the odour from the source to a sensor chamber. First results show that, although the second solution gives more accurate recognition results, the static operation could be sufficient for the online odour monitoring (Nicolas et al., 2000). However, the influence of air movement on the heated sensors sometimes disturbs the recognition, and a sensor chamber, or at least a shelter, seems always more adequate than a true open system. 
- The control of the temperature and the humidity of the gas and the thermo-regulation of the sensor chamber don't seem essential, even for outdoor operation. Some arguments may be put forward (Romain et al., 2000) :

- the fact that TGS sensors are heated maintains the working ambience in the sensor chamber inside an appropriate temperature and humidity range;

- the interference of ambient variables doesn't affect dramatically the odour recognition, as long as many different humidity and temperature conditions are included in the learning phase for a given odour;

Choice of a function adapted to classify the various sources so as to be able to make a decision in real time.

Beyond the graphical appearance of classification results, showing nice ellipses around well separated groups, the end user would like to have at his disposal a simple function allowing him to clearly evaluate the membership to a given group, in order to make a decision.

In this spirit, non-supervised analyses, such as PCA, provide basically a performance evaluation of the system during the development phase, but they do not really represent learning tools aiming at the calibration of a recognition model.

On the contrary, supervised analyses, such as Discriminant Analysis (DA), or some Neural Networks algorithms, should not be regarded as evaluation tools. The first reason is that they always give satisfactory results (in particular, a total of $50 \%$ of cases correctly classified seems a rather good result, but it is simply normal : such result is already reached with groups randomly created). Secondly, the model that they build is too specific to the operating conditions : it is valid for a given sensor array, a given gas flow rate and a given test protocol. Now, these fixed conditions are prevailing during the final utilisation phase of the apparatus in the field. There, the e-nose is not any more in the development phase, and it must provide a function of the type "yes or no", based on a preliminary training.

The various experiments conducted until now in the field show that the classification functions provided by the DA procedures are quite appropriate to make a reliable recognition in real time, when the system is developed. Those functions are linear combinations of signals, providing as many classification scores as identified groups. A particular case is assigned to the group for which it has the highest classification score. Their use is very simple, very convenient, and leads to unambiguous classification results.

Alternatively, artificial neural network with the Radial Basis Function (RBF) architecture may be used to handle also some "unknown" class (Horner, 1999).

Choice of the useful signal that the e-nose system must provide for the continuous monitoring of the odour intensity.

As long as only the classification of odour sources is concerned, the models calibrated during the learning phase, either by statistical methods (PCA, DA, ...), or by Artificial Neural Networks, could be included in the mobile detector for the on-line identification of unknown odours. When the identification is achieved, the estimation of the odour intensity by the system can be done by appropriate techniques. Whatever the final application of the monitoring may be -either assessing the nuisance or controlling an abatement system- the 
useful signal should be related to the monitored odour, and shouldn't be influenced by disturbing odours or ambient parameters.

For more than ten years, several techniques are proposed in the literature (Göpel et al, 1989). In short, they use either the signal of one sensor element, or a weighted signal of all elements, obtained by a suitable regression technique. Are those proposed techniques suited for the online monitoring of environmental odours?

Using one of the sensor elements, preferably that with the highest sensitivity towards the identified substance, is a rather easy solution. On the landfill area, we chose to monitor the signal of TGS2610 (more sensitive to a wide variety of combustible gas). The Kendall correlation coefficient between the TGS2610 resistance and the odour intensity class, as assessed by the field operator, is -0.87 and shows a rather strong relationship between the two variables. The model obtained by linear regression allows to predict the intensity class from the recorded sensor signal. The measured intensity class is correctly predicted in $65 \%$ of the 141 observations made on the landfill area.

However, the TGS2610 sensor is sensitive to both sources (fresh waste and biogas), and probably also to many other ones, so, its signal cannot be used to detect the rise of a particular odour among other ones. The procedure will thus always include two steps: a first identification of the odour by a classification technique, followed by the monitoring of the intensity of the global odour.

But the sensor varies also with ambient air temperature and humidity, and that is more awkward, since that kind of variation is unpredictable.

Such solution is thus applicable for "pure" substance, in the laboratory, but is not really suited to field applications, where background odours and variable ambient conditions affect the sensor response.

However, in the absence of other odours, the sole influence of ambient temperature and humidity could be taken into account (if they are measured) by a kind of "expert system". For example, the following rule can be applied :

If (the sensor resistance drops) AND (the humidity is stable or decreases) AND (the temperature is stable or decreases) THEN (there is probably an "odour event") OTHERWISE (any conclusion can't be drawn from the sensor resistance variation).

In this case, the system is able to detect the emergence of the odour only when the temperature and the humidity don't influence the sensor response. Such rule was experimented in Spring 1998 around the settling pond of a sugar factory and pointed out some "odour events", validated by the observation (Nicolas et al, 2000).

Anyway, using a weighted signal of all elements is more accurate and more "selective" to the monitored odour, i.e. minimises the cross-sensitivity (Hierold and Müller, 1989, Gall and Müller, 1989, Horner and Hierold, 1990).

If the classification model was calibrated by a supervised method, such as Discriminant Analysis (DA), the classification functions supplied as a standard result of such method can be used as "odour signal". Though they show very bad correlation with the measured intensity on the landfill, their selectivity to a given odour can be exploited for the continuous monitoring. Figure 1 shows such result : when moving away from the landfill gas extraction well, the classification function of the biogas decreases, when the one of fresh waste remains stable. 


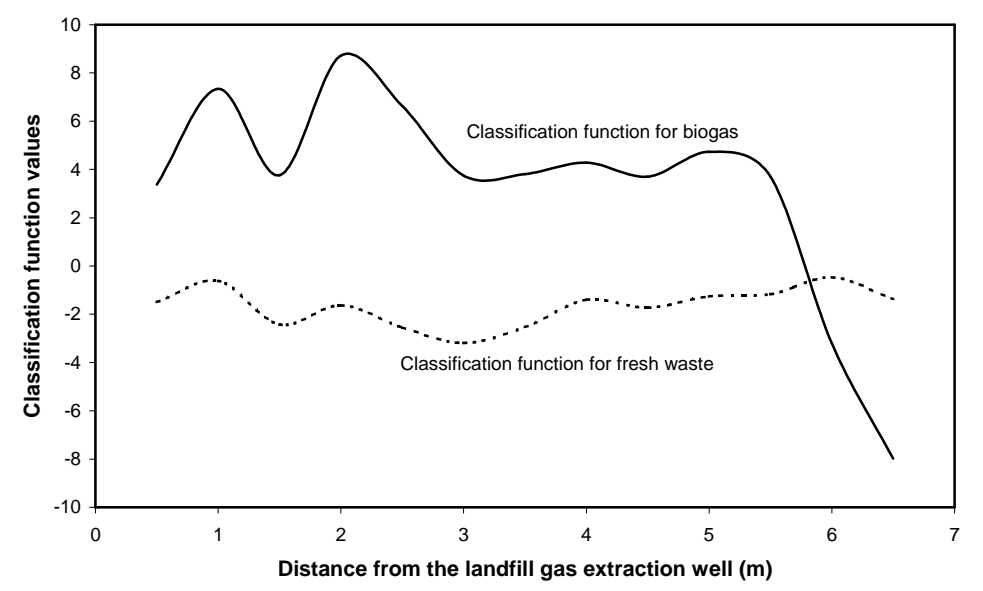

Figure 1 : Evolution of DA classification functions when moving away from the source

Different regression techniques may also be applied to predict the intensity of the odour generated on the landfill area. Multilinear Regression (MLR) on the 6 original measured sensor signals (resistances) provides a rather good model, which predicts an intensity value in agreement with the measured one in $67 \%$ of the cases. The resulting model, however, is a pure mathematical construction, which is convenient to predict intensity values inside the training sample, but which is less adapted to the prediction of new data.

Using the results of an unsupervised classification method, such as the factors supplied by a Principal Component Analysis (PCA), has a good chance to produce a more physical model, making more "sense" from a physical standpoint (Wise and Gallagher, 1998).

Indeed, the principal component regression (PCR) includes in the model the first principal component, which is already well correlated with the odour intensity, and the second one, which separates "biogas" from "fresh waste". Including the third one in the regression provides a model which predicts the measured intensity in $69 \%$ of the cases. Of course, the model converges towards the MLR one when the 3 remaining principal components are added. As this model MLR is worse than the model based on 3 principal components, it seems that some of the initial variables were not relevant for the prediction of the odour intensity.

Finally, Partial Least Squares regression (PLS), which captures the greatest amount of variance, like PCA, and also achieve correlation with the predictor variable (here the intensity), like MLR, will probably provide the most adapted model for the intensity prediction.

Indeed, testing PLS regression on the 141 observations on the landfill shows that the model provides $71 \%$ of intensity prevision in agreement with the measured one. That is a very good result, knowing that, in the $29 \%$ left, it remains probably a lot of errors of estimation due to the operator in the field. Moreover, like PCA, the PLS provides the classification of observations in two groups. Consequently, it should be used as sole tool, both to identify the source of the odour and to predicting its intensity. 


\section{References}

Gall, M. and Müller, R. 1989 Investigation of gas mixtures with different MOS Gas sensors with regard to pattern recognition. - Sensors and Actuators, 17, 583-586

Gardner, J.W. and Bertlett, P.N. 1999 Electronic Noses - Principles and applications - Oxford University Press - Oxford - 245 p.

Göpel, W., Hesse, J. and Zemel, J.N. (editors) 1989 Sensors - A comprehensive survey (Volume I) - VCH Verlagsgesellschaft mbH - Weinheim - 641 p.

Hierold, Chr. and Müller, R. 1989 Quantitative analysis of gas mixtures with non-selective gas sensors - Sensors and Actuators, 17, 587-592

Horner, G. and Hierold, Chr. 1990 Gas Analysis by Partial Model Building - Sensors and Actuators B, 2, 173-184

Horner, G. 1999 Qualitative and Quantitative Evaluation Methods for Sensorarrays Proceedings of ISOEN99 - Tübingen - September 20-22, 1999, 171-174

Nicolas, J., Romain, A.C., Wiertz, V., Maternova, J. and André, Ph. 1999 Utilisation élémentaire d'un réseau de capteurs "SnO2" pour la reconnaissance d'odeurs environnementales - Eurodeur99, Paris, 15-18 June 1999.

Nicolas, J., Romain, A.C., Wiertz, V., Maternova, J. and André, Ph. 2000 Using the classification model of an electronic nose to assign unknown malodours to environmental sources and to monitor them continuously. - Accepted for publication in Sensors and Actuators B.

Romain, A.C., Nicolas, J. and André, Ph. 1997 In situ measurement of olfactive pollution with inorganic semiconductors : limitations due to the influence of humidity and temperature Seminars in Food analysis, 2 (1997) 283-296.

Romain, A.C., Nicolas, J., Wiertz, V., Maternova, J. and André, Ph. 2000 Use of a simple tin oxide sensor array to identify five malodours collected in the field - Sensors and actuators B, 62, 73-79

Wise, B.M. and Gallagher, N.B. 1998 PLS_Toolbox 2.0 for use with MATLAB ${ }^{T M}$ - Eigen Research, Inc. - Manson - pp.320. 